\title{
Representations of Twisted Current Algebras
}

\author{
Michael Lau* \\ Université Laval \\ Département de mathématiques et de statistique \\ Québec, QC, Canada G1V 0A6 \\ Email: Michael.Lau@mat.ulaval.ca
}

\begin{abstract}
We use evaluation representations to give a complete classification of the finitedimensional simple modules of twisted current algebras. This generalizes and unifies recent work on multiloop algebras, current algebras, equivariant map algebras, and twisted forms.
\end{abstract}

MSC2010: primary 17B10; secondary 17B65, 17B05, 16W22

\section{Introduction}

Evaluation representations were introduced in the late 1980s to classify representations of loop algebras [7, 8, 17]. This powerful technique was later adapted to study representations of multiloop algebras [18, 2, 14] and various other generalizations and related Lie algebras. (See [12, 9, 13, 10], for instance.) Two very recent papers use evaluation representations to classify the finite-dimensional simple modules of equivariant map algebras and twisted forms [16, 15]. While these two families contains all the previously mentioned examples in the literature, twisted forms also include Margaux algebras (which are not equivariant map algebras) and equivariant map algebras include Onsager algebras (which are not twisted forms). Equivariant map algebras and twisted forms are both examples of twisted current algebras, fixed point subalgebras of tensor products of Lie algebras and associative algebras under a finite group action. The goal of this short paper is to classify the finite-dimensional simple modules of twisted current algebras up to isomorphism. This gives a unified perspective on evaluation representations, recovering the main results of [16] and [15] as special cases.

The work in [15] suggests that the common theme in the evaluation module approach is not the equivariant maps studied in [14, 16], but rather the invariants of current algebras under a finite group action. Let $\mathfrak{g}$ be a finite-dimensional simple Lie algebra over an algebraically closed field $k$ of characteristic zero, and let $X=\operatorname{Spec} S$ be a reduced affine $k$-scheme of finite type with coordinate algebra $S$. The $\mathfrak{g}$-valued regular functions on $X$ form a current Lie algebra $\mathfrak{g} \otimes_{k} S$ over $k$, with pointwise Lie bracket $[x \otimes r, y \otimes s]=[x, y] \otimes r s$, for $x, y \in \mathfrak{g}$ and $r, s \in S$. When a finite group $\Gamma$ acts by $k$-Lie

${ }^{*}$ Funding from the Natural Sciences and Engineering Research Council of Canada is gratefully acknowledged. 
algebra automorphisms on $\mathfrak{g} \otimes_{k} S$, the twisted current algebra is simply the fixed point subalgebra $\mathcal{L}=\left(\mathfrak{g} \otimes_{k} S\right)^{\Gamma}$ under the action of $\Gamma$.

Special cases include both the equivariant map algebras considered in [16] and the twisted forms of algebras studied in [15. Indeed, we obtain the equivariant map algebras when $\Gamma$ acts by automorphisms on both the Lie algebra $\mathfrak{g}$ and the $k$-algebra $S$, such that

$$
{ }^{\gamma}(x \otimes s)={ }^{\gamma} x \otimes{ }^{\gamma} s(x \in \mathfrak{g}, s \in S, \gamma \in \Gamma) .
$$

The twisted forms of [15] appear when $\Gamma$ acts on $\mathfrak{g} \otimes S$ via a group action on $S$ and a 1-cocycle $u: \Gamma \rightarrow \operatorname{Aut}_{S-\text { Lie }}\left(\mathfrak{g} \otimes_{k} S\right)$, such that

$$
{ }^{\gamma}(x \otimes s)=u_{\gamma}\left(x \otimes{ }^{\gamma} s\right)
$$

and the ring extension of $S$ over $S^{\Gamma}=\left\{s \in S:{ }^{\gamma} s=s\right.$ for all $\left.\gamma \in \Gamma\right\}$ is Galois.

The classification of finite-dimensional simple modules of a twisted current algebra $\mathcal{L}=\left(\mathfrak{g} \otimes_{k} S\right)^{\Gamma}$ begins with the observation that any such representation $\phi: \mathcal{L} \rightarrow$ End $V$ descends to a representation of $\mathcal{L} / \operatorname{ker} \phi$, a finite-dimensional reductive Lie algebra. Up to twisting by a character, the quotient map $\mathcal{L} \rightarrow \mathcal{L} / \operatorname{ker} \phi \subseteq$ End $V$ factors through the image of an evaluation homomorphism

$$
\mathrm{ev}_{\underline{M}}: \mathcal{L} \hookrightarrow \mathfrak{g} \otimes_{k} S \rightarrow\left(\mathfrak{g} \otimes_{k} S / M_{1}\right) \oplus \cdots \oplus\left(\mathfrak{g} \otimes_{k} S / M_{r}\right) \cong \mathfrak{g}^{\oplus r},
$$

for some family of maximal ideals $\underline{M}=\left\{M_{1}, \ldots, M_{r}\right\} \subseteq$ Max $S$. The isomorphism class of the representation $(V, \phi)$ is then determined (up to twisting by a character) by the choice of $\underline{M}$ and by the isomorphism class of $V$, viewed as a finite-dimensional simple $\operatorname{ev}_{\underline{M}}(\mathcal{L})$-module.

In the case of equivariant map algebras, the image of the evaluation homomorphism is a direct sum of isotropy subalgebras $\mathfrak{g}^{M_{i}}$ with respect to an action of subgroups $\Gamma^{M_{i}} \subseteq \Gamma$ fixing maximal ideals $M_{i}$. A priori, the definition of a twisted current algebra $\mathcal{L}=\left(\mathfrak{g} \otimes_{k} S\right)^{\Gamma}$ contains neither an action of $\Gamma$ on $\mathfrak{g}$ nor on $S$. However, the action of $\Gamma$ on $\mathfrak{g} \otimes_{k} S$ induces an action on the maximal ideals of $S$, and thus on $S$ itself. This lets us define a 1-cocycle $u: \Gamma \rightarrow \operatorname{Aut}_{S-L i e}\left(\mathfrak{g} \otimes_{k} S\right)$, so that the action of $\Gamma$ on $\mathfrak{g} \otimes_{k} S$ can be described as in the case of twisted forms: ${ }^{\gamma}(x \otimes s)=u_{\gamma}\left(x \otimes{ }^{\gamma} s\right)$. While the general ideas of the evaluation module approach remain the same, the fact that the extension $S / S^{\Gamma}$ need not be Galois (and thus $\mathcal{L} \otimes_{S^{\Gamma}} S$ need not be isomorphic to $\mathfrak{g} \otimes S$ ) invalidates some of the most important descent-based arguments in this context.

Instead, we adopt the approach presented in [16]. The major obstacle here is the reliance on an action of $\Gamma$ on the finite-dimensional Lie algebra $\mathfrak{g}$ to define equivariant map algebras. Such an action no longer exists in the context of twisted current algebras. We finesse this difficulty by using local twisted actions, an idea motivated by ideas from affine group schemes. If $\Gamma \subseteq \operatorname{Aut}_{S-L i e}\left(\mathfrak{g} \otimes_{k} S\right)$, then $\Gamma$ is an affine group scheme over $S$, with $\Gamma(A) \subseteq \operatorname{Aut}_{A-L i e}(\mathfrak{g}(A))$ given by the $A$-linear extension of the $\Gamma$-action on $\mathfrak{g} \otimes_{k} S$ to $\mathfrak{g}(A)=\left(\mathfrak{g} \otimes_{k} S\right) \otimes_{S} A$. In particular, if $A=S / M$ for some maximal ideal $M$ of $S$, then $\mathfrak{g}(A) \cong \mathfrak{g}$, and we have a group homomorphism ev $M$ associated to each $M \in \operatorname{Max} S$ :

$$
\begin{aligned}
\Gamma & \stackrel{\mathrm{ev}_{M}}{\longrightarrow} \Gamma(S / M) \subseteq \operatorname{Aut}_{k-L i e}(\mathfrak{g}) \\
\alpha & \longmapsto \alpha(M) .
\end{aligned}
$$


When working with twisted current algebras, the action of $\Gamma$ is only $k$-linear, in general, and each evaluation map $\mathrm{ev}_{M}$ gives a twisted action on $\mathfrak{g}$ :

$$
(\alpha \beta)(M)=\alpha(M)^{\alpha} \beta(M) \quad\left(\alpha, \beta \in \operatorname{Aut}_{k-\text { Lie }}\left(\mathfrak{g} \otimes_{k} S\right), M \in \operatorname{Max} S\right) .
$$

See Section 3 for details. This local twisted action turns out to be the crucial ingredient to adapt the proofs of [16] to the framework of twisted forms, and more generally, to twisted current algebras.

The methods in this paper can also be used to study the abelian tensor category $\mathcal{C}$ of finite-dimensional modules of twisted current algebras, a topic we plan to explore in a future paper [1]. In particular, this will classify the blocks of $\mathcal{C}$, and consequently the blocks of twisted forms of algebras.

\section{Twisted current algebras}

Throughout this paper, $k$ will denote an algebraically closed field of characteristic zero, $\mathfrak{g}$ will be a finite-dimensional simple Lie algebra over $k, X$ will be a reduced affine $k$-scheme of finite type, and the (untwisted) current algebra $\mathfrak{M}=\mathfrak{M}(X, \mathfrak{g})$ will be the space of $\mathfrak{g}$-valued regular functions on $X$. This is a Lie algebra under the pointwise Lie bracket $[f, g](M)=[f(M), g(M)]$, for all $f, g \in \mathfrak{M}$ and $k$-rational points $M \in X$. Let $\Gamma$ be a finite group acting on $\mathfrak{M}$ by $k$-Lie algebra automorphisms. The subalgebra of $\Gamma$-invariants,

$$
\mathfrak{M}(X, \mathfrak{g})^{\Gamma}=\left\{f \in \mathfrak{M}:{ }^{\gamma} f=f \text { for all } \gamma \in \Gamma\right\},
$$

is called the twisted current algebra associated with $\mathfrak{M}$ and $\Gamma$.

If $S$ is the coordinate algebra of $X$, we denote by $s(M) \in k$ the residue class of $s \in S$ modulo any maximal ideal ( $k$-rational point) $M \in \operatorname{Max} S$ :

$$
s(M)+M=s+M \in S / M \cong k .
$$

The twisted current algebra $\mathfrak{M}(X, \mathfrak{g})^{\Gamma}$ is isomorphic to, and will henceforth be identified with, the Lie algebra of fixed points

$$
\mathcal{L}=\left(\mathfrak{g} \otimes_{k} S\right)^{\Gamma}=\left\{z \in \mathfrak{g} \otimes_{k} S:{ }^{\gamma} z=z\right\}
$$

where the action of $\Gamma$ on $\mathfrak{g} \otimes_{k} S$ is obtained by identifying $\mathfrak{g} \otimes_{k} S$ with $\mathfrak{M}(X, \mathfrak{g})$ via

$$
\left(\sum x_{i} \otimes_{k} s_{i}\right)(M):=\sum s_{i}(M) x_{i}
$$

for all $x_{i} \in \mathfrak{g}, s_{i} \in S$, and $k$-rational points $M$ in $X$. Twisted current algebras are then precisely the $\Gamma$-fixed subalgebras of $k$-Lie algebras $\mathfrak{g} \otimes_{k} S$, where $S$ is a unital, commutative, associative, and reduced $k$-algebra of finite type. However, note that while the untwisted current algebra $\mathfrak{g} \otimes_{k} S$ is naturally a Lie algebra over the base ring $S$, the action of $\Gamma$, being only $k$-linear, defines only a $k$-Lie algebra structure on the twisted current algebra $\mathcal{L}=\left(\mathfrak{g} \otimes_{k} S\right)^{\Gamma}$. 
Proposition 2.2 Let $\mathcal{L}=\left(\mathfrak{g} \otimes_{k} S\right)^{\Gamma}$ be the twisted current algebra associated to the action of $\Gamma$ on $\mathfrak{g} \otimes_{k} S$. Then there is a group action of $\Gamma$ on $S$ and a crossed homomorphism $u: \Gamma \rightarrow \operatorname{Aut}_{S-\text { Lie }}\left(\mathfrak{g} \otimes_{k} S\right)$ such that

$$
\gamma_{\left(x \otimes_{k} s\right)}=u_{\gamma}\left(x \otimes_{k}^{\gamma} s\right)
$$

for all $\gamma \in \Gamma, x \in \mathfrak{g}$, and $s \in S$.

Proof Let $\gamma \in \Gamma$, and let $P$ be an ideal of $S$. Since $\Gamma$ acts by automorphisms on $\mathfrak{g} \otimes_{k} S$, the image $\gamma\left(\mathfrak{g} \otimes_{k} P\right)$ of $\left(\mathfrak{g} \otimes_{k} P\right)$ under the action of $\gamma$ is an ideal of $\mathfrak{g} \otimes_{k} S$. Every such ideal is of the form $\mathfrak{g} \otimes_{k} I$ for some ideal $I$ of $S$, so

$$
{ }^{\gamma}\left(\mathfrak{g} \otimes_{k} P\right)=\mathfrak{g} \otimes_{k}{ }^{\gamma} P,
$$

for some ideal ${ }^{\gamma} P \subseteq S$. Restricted to the set Max $S$ of maximal ideals of $S$, this gives a group action of $\Gamma$ on $\operatorname{Max} S$.

Since $S$ is reduced and of finite type over an algebraically closed field, the Jacobson radical of $S$ is trivial. The action of $\Gamma$ on Max $S$ thus determines an action of $\Gamma$ on $S$ by $k$-algebra automorphisms. Explicitly, the image ${ }^{\gamma} s$ of each $s \in S$ under $\gamma \in \Gamma$ is determined by its reduction modulo the maximal ideals $M \in \operatorname{Max} S$ :

$$
{ }^{\gamma} s(M)=s\left(\gamma^{-1} M\right) .
$$

For each $\gamma \in \Gamma$, let $u_{\gamma}: \mathfrak{g} \otimes_{k} S \rightarrow \mathfrak{g} \otimes_{k} S$ be the $k$-linear map defined by

$$
u_{\gamma}(x \otimes s)={ }^{\gamma}\left(x \otimes \gamma^{-1} s\right),
$$

for all $x \otimes s \in \mathfrak{g} \otimes_{k} S$. It is straightforward to verify that $u_{\gamma}$ is a $k$-Lie algebra automorphism of $\mathfrak{g} \otimes_{k} S$.

We now show that the automorphism $u_{\gamma}$ is actually $S$-linear. Consider $r, s \in S$, $x \in \mathfrak{g}$, and $\gamma \in \Gamma$. For each $P \in \operatorname{Max} S$, there exists $t \in \gamma^{-1} P$ such that $s=s\left(\gamma^{-1} P\right)+t$. Therefore,

$$
\begin{aligned}
{ }^{\gamma}(x \otimes r s)(P) & ={ }^{\gamma}\left(x \otimes(r)\left(s\left(^{\gamma^{-1}} P\right)\right)(P)+{ }^{\gamma}(x \otimes r t)(P)\right. \\
& =s\left({ }^{\gamma^{-1}} P\right)^{\gamma}(x \otimes r)(P)+{ }^{\gamma}(x \otimes r t)(P) .
\end{aligned}
$$

But $r t \in \gamma^{-1} P$, so ${ }^{\gamma}(x \otimes r t) \in^{\gamma}\left(\mathfrak{g} \otimes \gamma^{-1} P\right)=\mathfrak{g} \otimes P$. Thus ${ }^{\gamma}(x \otimes r t)(P)=0$ and

$$
\left.\gamma_{(x} \otimes r s\right)(P)={ }^{\gamma} s(P)^{\gamma}(x \otimes r)(P) .
$$

Expanding with respect to a $k$-basis $\left\{x_{i}\right\}$ of $\mathfrak{g}$, we write

$$
{ }^{\gamma}(x \otimes r s)=\sum_{i} x_{i} \otimes m_{i} \quad \text { and } \quad \gamma(x \otimes r)=\sum_{i} x_{i} \otimes r_{i}
$$

for some $m_{i}, r_{i} \in S$. Thus ${ }^{\gamma}(x \otimes r s)(P)=\sum_{i} m_{i}(P) x_{i}$ and ${ }^{\gamma} s(P)^{\gamma}(x \otimes r)(P)=$ $\gamma_{s}(P) \sum r_{i}(P) x_{i}$, so $m_{i}(P)={ }^{\gamma} s(P) r_{i}(P)$ for all $P \in \operatorname{Max} S$ and for all $i$. Since the Jacobson radical of $S$ is zero, $m_{i}={ }^{\gamma} s r_{i}$ for all $i$, and

$$
{ }^{\gamma}(x \otimes r s)={ }^{\gamma} s^{\gamma}(x \otimes r) .
$$


In particular,

$$
\begin{aligned}
u_{\gamma}(x \otimes r s) & =\gamma\left(x \otimes \gamma^{-1}(r s)\right) \\
& =\gamma\left(x \otimes \gamma^{-1} r^{\gamma^{-1}} s\right) \\
& =s^{\gamma}\left(x \otimes \gamma^{-1} r\right) \\
& =s u_{\gamma}(x \otimes r),
\end{aligned}
$$

so $u_{\gamma}$ is $S$-linear.

The finite group $\Gamma$ acts on the group $\operatorname{Aut}_{S-L i e}\left(\mathfrak{g} \otimes_{k} S\right)$ of $S$-Lie algebra automorphisms in the usual way:

$$
{ }^{\gamma} \phi=(1 \otimes \gamma) \circ \phi \circ\left(1 \otimes \gamma^{-1}\right)
$$

where $(1 \otimes \gamma)(x \otimes s)=x \otimes{ }^{\gamma} s$ for all $\gamma \in \Gamma, \phi \in \operatorname{Aut}_{S-L i e}\left(\mathfrak{g} \otimes_{k} S\right)$, and $x \otimes s \in \mathfrak{g} \otimes_{k} S$. Therefore,

$$
\begin{aligned}
u_{\gamma}^{\gamma} u_{\eta}(x \otimes s) & =u_{\gamma}\left((1 \otimes \gamma)^{\eta}\left(x \otimes \eta^{-1} \gamma^{-1} s\right)\right) \\
& =\gamma\left(\left(1 \otimes \gamma^{-1}\right) \circ(1 \otimes \gamma)^{\eta}\left(x \otimes \eta^{-1} \gamma^{-1} s\right)\right) \\
& \left.=\gamma \eta\left(x \otimes \eta^{-1} \gamma^{-1} s\right)\right) \\
& =u_{\gamma \eta}(x \otimes s),
\end{aligned}
$$

for all $x \otimes s \in \mathfrak{g} \otimes S$ and $\gamma, \eta \in \Gamma$. Thus $u_{\gamma \eta}=u_{\gamma}^{\gamma} u_{\eta}$, and $u: \Gamma \rightarrow \operatorname{Aut}_{S-L i e}\left(\mathfrak{g} \otimes_{k} S\right)$ is a crossed homomorphism.

Let $R=S^{\Gamma}=\left\{s \in S: \gamma_{s}=s\right.$ for all $\left.\gamma \in \Gamma\right\}$ be the subalgebra of $\Gamma$-invariants in $S$. Then $r \mathcal{L} \subseteq \mathcal{L}$ for all $r \in R$, so $\mathcal{L}$ has the structure of a Lie algebra over $R$, and it follows that all maximal $k$-Lie algebra ideals $\mathcal{I}$ of $\mathcal{L}$ which do not contain the derived subalgebra $[\mathcal{L}, \mathcal{L}]$ are actually $R$-Lie algebra ideals of $\mathcal{L}$. See [16, Lemma 4.16] or [15, Lemma 2.7] for details. Note that these are precisely the ideals $\mathcal{I}$ of $\mathcal{L}$ for which the quotient $\mathcal{L} / \mathcal{I}$ is a simple Lie algebra. We denote this set of $R$-ideals by $\operatorname{Max} \mathcal{L}$. For each $M \in \operatorname{Max} S$, we write $\Gamma^{M}=\left\{\gamma \in \Gamma:{ }^{\gamma} M=M\right\}$ for the isotropy subgroup of $M$, and we denote by $\psi(M): \mathfrak{g} \rightarrow \mathfrak{g}$ the map $\psi(M) x=(\psi(x \otimes 1))(M)$ for each $\psi \in$ Aut $_{k-\text { Lie }}(\mathfrak{g} \otimes S)$ and $x \in \mathfrak{g}$. The finite-dimensional Lie algebra $\mathfrak{g}^{M}=\left\{x \in \mathfrak{g}: \gamma(M) x=x\right.$ for all $\left.\gamma \in \Gamma^{M}\right\}$ will reappear later in this section as the image of the evaluation map $\operatorname{ev}_{M}: \mathcal{L} \rightarrow \mathfrak{g}$ sending an element $\alpha \in \mathcal{L}$ to $\alpha(M)$, as defined in (2.1).

Lemma 2.7 For each $R$-ideal $\mathcal{M}$ of $\mathcal{L}$, let $I(\mathcal{M})=\{r \in S: r \mathcal{L} \subseteq \mathcal{M}\}$. Then $I(\mathcal{M}) \in \operatorname{Max} R$ for all $\mathcal{M} \in \operatorname{Max} \mathcal{L}$.

Proof Suppose $\mathcal{M} \in \operatorname{Max} \mathcal{L}$. Then $\mathcal{L} / \mathcal{M}$ is a simple module for the (countable dimensional) Lie algebra $\mathcal{L}$, and $I(\mathcal{M})$ is the kernel of the map $m: R \rightarrow \operatorname{End}_{\mathcal{L}-\bmod }(\mathcal{L} / \mathcal{M})$, $m: r \mapsto m_{r}$, where $m_{r}: \mathcal{L} / \mathcal{M} \rightarrow \mathcal{L} / \mathcal{M}, \bar{x} \mapsto \overline{r x}$. By Schur's Lemma, we see that each $m_{r}$ is a scalar multiplication, and $I(\mathcal{M})=$ ker $m$ is a maximal ideal of $R$. 
Remark 2.8 If the extension $S / R$ is Galois, then the map $I: \operatorname{Max} \mathcal{L} \rightarrow \operatorname{Max} R$ is a bijection, as follows from [15, Theorem 2.9]. In the context of twisted current algebras, this is not true in general. For example, let $\mathfrak{g}=\mathfrak{s l}_{2}(k), S=k \oplus k \oplus k$, and $\Gamma$ the Klein-IV group $\mathbb{Z}_{2} \oplus \mathbb{Z}_{2}$. Choose Chevalley generators $e, f, h$ for $\mathfrak{s l}_{2}(k)$. Let $(a, b) \in \Gamma$ act on $x \otimes s$ for $x \in \mathfrak{g}$ and $s=\left(s_{1}, s_{2}, s_{3}\right) \in S$ by

$$
(a, b) \cdot(x \otimes s)=\sigma_{1}^{a} \circ \sigma_{2}^{b}(x) \otimes \gamma^{a+b}(s),
$$

where $\sigma_{\ell}(e)=(-1)^{\ell} f, \sigma_{\ell}(f)=(-1)^{\ell} e$, and $\sigma_{\ell}(h)=-h$ for $\ell=1,2$, and $\gamma\left(s_{1}, s_{2}, s_{3}\right)=$ $\left(s_{2}, s_{1}, s_{3}\right)$. The maximal ideal $J=\{(a, a, 0): a \in k\}$ of $R=\{(a, a, b): a, b \in k\}$ has the property that $J \mathcal{L}=\mathcal{L}$, where $\mathcal{L}=\left(\mathfrak{g} \otimes_{k} S\right)^{\Gamma}=\operatorname{Span}_{k}\left\{h \otimes_{k}(1,-1,0)\right\}$, so there is no maximal ideal $\mathcal{M} \subset \mathcal{L}$ for which $I(\mathcal{M})=J$.

We now prove the following proposition using a modification of the argument in 16 , Proposition 5.2]. Because the proposition is nontrivial and plays an important role in what follows, we give a detailed proof in the context of twisted current algebras for completeness.

Proposition 2.9 Let $\mathcal{M}_{1}, \ldots, \mathcal{M}_{s} \in \operatorname{Max} \mathcal{L}$ be distinct ideals for which $I\left(\mathcal{M}_{1}\right)=\cdots=$ $I\left(\mathcal{M}_{s}\right)$. Then there is a natural Lie algebra epimorphism $\mathfrak{g}^{M} \rightarrow \mathcal{L} / \mathcal{M}_{1} \times \cdots \times \mathcal{L} / \mathcal{M}_{s}$ for any $M \in \operatorname{Max} S$ lying over $I=I\left(\mathcal{M}_{1}\right)$.

Proof Since $\Gamma$ fixes the elements of $R$, it leaves $I$ and $I S$ (setwise) invariant. The exact sequence $0 \rightarrow I S \rightarrow S \rightarrow S / I S \rightarrow 0$ induces an exact sequence of $\Gamma$-modules:

$$
0 \rightarrow \mathfrak{g} \otimes_{k} I S \rightarrow \mathfrak{g} \otimes_{k} S \rightarrow \mathfrak{g} \otimes_{k} S / I S \rightarrow 0 .
$$

Taking $\Gamma$-invariants is an exact functor, so

$$
\mathcal{L} / I \mathcal{L}=\left(\mathfrak{g} \otimes_{k} S\right)^{\Gamma} /\left(\mathfrak{g} \otimes_{k} I S\right)^{\Gamma} \cong\left(\mathfrak{g} \otimes_{k} S / I S\right)^{\Gamma} .
$$

By definition, $I \mathcal{L} \subseteq \mathcal{M}_{1} \cap \cdots \cap \mathcal{M}_{s}$, so we have a surjective homomorphism

$$
\psi:\left(\mathfrak{g} \otimes_{k} S / I S\right)^{\Gamma} \rightarrow \mathcal{L} /\left(\mathcal{M}_{1} \cap \cdots \cap \mathcal{M}_{s}\right) .
$$

The extension $S / R$ is integral [4, $\S 1.9$ Proposition 22], so $\Gamma$ acts transitively on the set of (finitely many) maximal ideals $M_{1}, \ldots, M_{r} \in \operatorname{Max} S$ lying over $I \in \operatorname{Max} R$, and $\sqrt{I S}=\bigcap_{i=1}^{r} M_{i}$ by [4, $\S 2.1$ Proposition 1 and $\S 2.2$ Théorème 2]. The action of $\Gamma$ on $\mathfrak{g} \otimes S / \sqrt{I S}$ thus transitively permutes the summands $\mathfrak{g} \otimes_{k} S / M_{i}$ in the direct sum

$$
\bigoplus_{i}\left(\mathfrak{g} \otimes_{k} S / M_{i}\right) \cong \mathfrak{g} \otimes_{k}\left(S / \cap M_{i}\right) \cong \mathfrak{g} \otimes_{k}(S / \sqrt{I S})
$$

For any $M=M_{i}$ lying over $I$, we see that projection onto the $i$ th component $\mathfrak{g} \otimes_{k} S / M_{i}$ induces an isomorphism of $\Gamma$-invariants: $(\mathfrak{g} \otimes S / \sqrt{I S})^{\Gamma} \stackrel{\pi}{\rightarrow}\left(\mathfrak{g} \otimes_{k} S / M\right)^{\Gamma^{M}}$, since taking $\Gamma$-invariants is an exact functor.

The evaluation map

$$
\begin{aligned}
\mathrm{ev}_{M}:\left(\mathfrak{g} \otimes_{k} S / M\right)^{\Gamma^{M}} & \rightarrow \mathfrak{g} \\
\sum x_{i} \otimes\left(s_{i}+M\right) & \mapsto \sum s_{i}(M) x_{i}
\end{aligned}
$$


has image contained in $\mathfrak{g}^{M}$. Indeed, for any $\sum x_{i} \otimes\left(s_{i}+M\right) \in\left(\mathfrak{g} \otimes_{k} S / M\right)^{\Gamma}$ and $\gamma \in \Gamma^{M}$, we see that $s_{i}(M)=s_{i}+m_{i}$ for some $m_{i} \in M$ and

$$
\begin{aligned}
\gamma(M)\left(\operatorname{ev}_{M}\left(\sum x_{i} \otimes\left(s_{i}+M\right)\right)\right) & =\gamma(M) \sum s_{i}(M) x_{i} \\
& =\sum \gamma\left(x_{i} \otimes s_{i}(M)\right)(M) \\
& ={ }^{\gamma}\left(\sum x_{i} \otimes\left(s_{i}+m_{i}\right)\right)(M) \\
& =\operatorname{ev}_{M}\left(\sum x_{i} \otimes s_{i}\right)+\gamma\left(\sum x_{i} \otimes m_{i}\right)(M) .
\end{aligned}
$$

Since $\gamma \in \Gamma^{M}$, we see that ${ }^{\gamma}\left(\sum x_{i} \otimes m_{i}\right) \in^{\gamma}\left(\mathfrak{g} \otimes_{k} M\right)=\mathfrak{g} \otimes_{k} M$, so ${ }^{\gamma}\left(\sum x_{i} \otimes m_{i}\right)(M)=$ $0, \operatorname{ev}_{M}\left(\sum x_{i} \otimes\left(s_{i}+M\right)\right)$ is fixed under $\gamma(M)$, and the image of $\mathrm{ev}_{M}$ is contained in $\mathfrak{g}^{M}$. It is then straightforward to verify that $\mathrm{ev}_{M}:\left(\mathfrak{g} \otimes_{k} S / M\right)^{\Gamma^{M}} \rightarrow \mathfrak{g}^{M}$ is a Lie algebra isomorphism.

Let $\alpha=\sum x_{i} \otimes s_{i} \in\left(\mathfrak{g} \otimes_{k} \sqrt{I S} / I S\right)^{\Gamma}$. The ideal $\mathcal{I} \subseteq\left(\mathfrak{g} \otimes_{k} S / I S\right)^{\Gamma}$ generated by $\alpha$ is contained in $\left(\mathfrak{g} \otimes_{k} \sqrt{I S} / I S\right)^{\Gamma}$, and is thus nilpotent. The $\mathcal{M}_{i}$ are distinct, so by the Chinese remainder theorem, the Lie algebra

$$
\mathcal{L} /\left(\mathcal{M}_{1} \cap \cdots \cap \mathcal{M}_{s}\right) \cong \mathcal{L} / \mathcal{M}_{1} \times \cdots \times \mathcal{L} / \mathcal{M}_{s}
$$

is semisimple. Therefore, $\mathcal{I} \subseteq \operatorname{ker} \psi$, and the map $\psi$ descends to a surjection

$$
\left(\mathfrak{g} \otimes_{k} S / I S\right)^{\Gamma} /\left(\mathfrak{g} \otimes_{k} \sqrt{I S} / I S\right)^{\Gamma} \rightarrow \mathcal{L} / \mathcal{M}_{1} \times \cdots \times \mathcal{L} / \mathcal{M}_{s} .
$$

Since taking $\Gamma$-invariants is an exact functor, we have

$$
\left(\mathfrak{g} \otimes_{k} S / \sqrt{I S}\right)^{\Gamma}=\left(\mathfrak{g} \otimes_{k} S / I S\right)^{\Gamma} /\left(\mathfrak{g} \otimes_{k} \sqrt{I S} / I S\right)^{\Gamma}=\left(\mathfrak{g} \otimes_{k} S / I S\right)^{\Gamma} /\left(\mathfrak{g} \otimes_{k} \sqrt{I S} / I S\right)^{\Gamma},
$$

and the map $\psi$ thus gives a surjective Lie algebra homomorphism

$$
\mathfrak{g}^{M} \cong\left(\mathfrak{g} \otimes_{k} S / M\right)^{\Gamma^{M}} \cong\left(\mathfrak{g} \otimes_{k} S / \sqrt{I S}\right)^{\Gamma} \stackrel{\psi}{\rightarrow} \mathcal{L} / \mathcal{M}_{1} \times \cdots \times \mathcal{L} / \mathcal{M}_{s} .
$$

\section{Classification of finite-dimensional simple modules}

We maintain the notation of the previous section: $k$ will be an algebraically closed field of characteristic zero, $S$ a unital, commutative, associative, and reduced $k$-algebra of finite type, $\mathfrak{g}$ a finite-dimensional simple $k$-Lie algebra, and $\Gamma$ a finite group acting by $k$-Lie algebra automorphisms on $\mathfrak{g} \otimes_{k} S$. By Proposition 2.2 , there then exists an action of $\Gamma$ on $S$ and a 1-cocycle $u: \Gamma \rightarrow \operatorname{Aut}_{S-L i e}\left(\mathfrak{g} \otimes_{k} S\right)$ defining the action of $\Gamma$ : $\gamma(x \otimes s)=u_{\gamma}\left(x \otimes^{\gamma} s\right)$, for all $\gamma \in \Gamma$ and $x \otimes s \in \mathfrak{g} \otimes_{k} S$. The action of $\Gamma$ is $R=S^{\Gamma}$-linear, and the associated twisted current algebra is the $k$-Lie algebra $\mathcal{L}=\left(\mathfrak{g} \otimes_{k} S\right)^{\Gamma}$. All

modules (representations) will be finite dimensional over $k$. Unless explicitly indicated otherwise, $\otimes$ will denote the tensor product $\otimes_{k}$ taken over $k$. 
Theorem 3.1 Let $\phi: \mathcal{L} \rightarrow \operatorname{End}_{k}(V)$ be an irreducible finite-dimensional representation of $\mathcal{L}$. Then there exist maximal ideals $M_{1}, \ldots, M_{r} \in M a x S$ in distinct $\Gamma$-orbits, finitedimensional simple $\mathfrak{g}^{M_{i}}$-modules $\left(V_{i}, \phi_{i}\right)$ (for $\left.i=1, \ldots, r\right)$, and a (possibly trivial) 1dimensional $\mathcal{L}$-module $W$, such that $V \cong W \otimes V_{1} \otimes \cdots \otimes V_{r}$, with $\mathcal{L}$-action defined by $\phi_{i} \circ \mathrm{ev}_{M_{i}}$ on each $V_{i}$.

Proof Since $\mathcal{L} / \operatorname{ker} \phi$ has a faithful finite-dimensional irreducible representation, it is reductive with centre $Z$ of dimension at most 1. See [5, $\$ 6$ Exercice 20] or [11, Exercise 12.4], for instance. Hence,

$$
\mathcal{L} / \operatorname{ker} \phi \cong Z \times \mathcal{L} / \mathcal{M}_{1} \times \cdots \times \mathcal{L} / \mathcal{M}_{\ell},
$$

for distinct maximal ideals $\mathcal{M}_{1}, \ldots, \mathcal{M}_{\ell} \subseteq \mathcal{L}$ where $\mathcal{L} / \mathcal{M}_{i}$ is a finite-dimensional simple Lie algebra for each $i=1, \ldots, \ell$. Therefore, $V$ is isomorphic to $W \otimes U_{1} \otimes \cdots \otimes U_{\ell}$, where $W$ is a 1-dimensional $\mathcal{L}$-module (trivial if $Z$ is zero), and $U_{i}$ is a finite-dimensional simple $\mathcal{L} / \mathcal{M}_{i}$-module, on which $\mathcal{L}$ acts by pulling back the action of $\mathcal{L} / \mathcal{M}_{i}$.

Suppose $I\left(\mathcal{M}_{i_{1}}\right)=\cdots=I\left(\mathcal{M}_{i_{s}}\right)$ for some $i_{1}, \ldots, i_{s} \in\{1, \ldots, r\}$. If $M$ is a maximal ideal of $S$ lying over $I\left(\mathcal{M}_{i_{1}}\right) \subset R$, then Proposition 2.9 gives an epimorphism $\psi: \mathfrak{g}^{M} \rightarrow$ $\mathcal{L} / \mathcal{M}_{i_{1}} \times \cdots \times \mathcal{L} / \mathcal{M}_{i_{s}}$. The kernel of the composition $\psi$ oev $_{M_{i}}: \mathcal{L} \rightarrow \mathcal{L} / \mathcal{M}_{i_{1}} \times \cdots \times \mathcal{L} / \mathcal{M}_{i_{s}}$ is contained in $\mathcal{M}_{i_{1}} \cap \cdots \cap \mathcal{M}_{i_{s}}$, so the irreducible representation

$$
\mathcal{L} \rightarrow \mathcal{L} / \mathcal{M}_{i_{1}} \times \cdots \times \mathcal{L} / \mathcal{M}_{i_{s}} \rightarrow \operatorname{End}\left(U_{i_{1}} \otimes \cdots \otimes U_{i_{s}}\right)
$$

factors through an irreducible representation of $\mathfrak{g}^{M}$ :

$$
\mathcal{L} \stackrel{\mathrm{ev}_{M}}{\rightarrow} \mathfrak{g}^{M} \stackrel{\psi}{\rightarrow} \mathcal{L} / \mathcal{M}_{i_{1}} \times \cdots \times \mathcal{L} / \mathcal{M}_{i_{s}} \rightarrow \operatorname{End}\left(U_{i_{1}} \otimes \cdots \otimes U_{i_{s}}\right) .
$$

Let $I_{1}, \ldots, I_{r} \in \operatorname{Max} R$ be the distinct ideals in the set $\left\{I\left(\mathcal{M}_{j}\right): j=1, \ldots, \ell\right\}$. For each $i=1, \ldots, r$, let $M_{i}$ be any maximal ideal of $S$ lying over $I_{i}$. Since the group $\Gamma$ acts on the fibres in Max $S$ over each ideal in Max $R$, the $M_{i}$ belong to distinct $\Gamma$-orbits. By the discussion above, the $\mathcal{L}$-module $V$ is thus isomorphic to $W \otimes V_{1} \otimes \cdots \otimes V_{r}$ for finite-dimensional simple $\mathfrak{g}^{M_{i}}$-modules $V_{i}=U_{i_{1}} \otimes \cdots \otimes U_{i_{n_{i}}}$, where $M_{i}$ lies over $I\left(\mathcal{M}_{i_{j}}\right)$ and $U_{i_{j}}$ is the $\mathcal{L} / \mathcal{M}_{i_{j}}$-module in the factorization $V \cong W \otimes U_{1} \otimes \cdots U_{\ell}$.

We now prove the converse of Theorem 3.1

Theorem 3.2 Let $M_{1}, \ldots, M_{r} \in$ Max $S$ be representatives of distinct $\Gamma$-orbits. Let $\left(V_{i}, \phi_{i}\right)$ be a finite-dimensional irreducible representation of $\mathfrak{g}^{M_{i}}$ for $i=1, \ldots, r$, and suppose that $(W, \rho)$ is a (possibly trivial) 1-dimensional module for the Lie algebra $\mathcal{L}$. Then $V=W \otimes V_{1} \otimes \cdots \otimes V_{r}$ is a finite-dimensional simple module for $\mathcal{L}$ with action $\phi_{i} \circ \mathrm{ev}_{M_{i}}$ on each $V_{i}$.

Proof By construction, $V$ is a simple module for $Z \times \mathfrak{g}^{M_{1}} \times \cdots \times \mathfrak{g}^{M_{r}}$, where $Z=\operatorname{ker} \rho$. It thus suffices to show that the map

$$
\left(p, \mathrm{ev}_{M}\right): \mathcal{L} \rightarrow Z \times \mathfrak{g}^{M_{1}} \times \cdots \times \mathfrak{g}^{M_{r}}
$$


is surjective, where $p$ is the canonical projection $\mathcal{L} \rightarrow \mathcal{L} / \mathcal{M}_{0}$ and $\operatorname{ev}_{M}=\left(\operatorname{ev}_{M_{1}}, \ldots, \mathrm{ev}_{M_{r}}\right)$. Let $I_{i}=M_{i} \cap R$ for $i=1, \ldots, r$. Since $S / R$ is integral and the $M_{i}$ belong to distinct $\Gamma$-orbits, the $I_{i}$ are distinct maximal ideals of $R$. Thus $I_{i}+I_{j}=R$ for all $i \neq j$, from which it follows that $I_{i} S+I_{j} S=S$ whenever $i \neq j$. Hence

$$
S / \bigcap_{i=1}^{r} I_{i} S \cong\left(S / I_{1} S\right) \oplus \cdots \oplus\left(S / I_{r} S\right),
$$

and the canonical map

$$
\pi: \mathfrak{g} \otimes S \rightarrow \mathfrak{g} \otimes S /\left(\mathfrak{g} \otimes \bigcap_{i=1}^{r} I_{i} S\right) \cong \bigoplus_{i=1}^{r}\left(\mathfrak{g} \otimes\left(S / I_{i} S\right)\right)
$$

is a surjection. Taking $\Gamma$-invariants is an exact functor, so the restriction of $\pi$ to $\mathcal{L}$ is also surjective:

$$
\pi: \quad \mathcal{L}=(\mathfrak{g} \otimes S)^{\Gamma} \rightarrow\left(\bigoplus_{i=1}^{r}\left(\mathfrak{g} \otimes S / I_{i} S\right)\right)^{\Gamma} .
$$

Since $I_{i} S$ is $\Gamma$-stable, $\left.\left(\bigoplus_{i=1}^{r}\left(\mathfrak{g} \otimes S / I_{i} S\right)\right)^{\Gamma}=\bigoplus_{i=1}^{r}\left(\mathfrak{g} \otimes S / I_{i} S\right)\right)^{\Gamma}$. By $(\underline{2.10}),(\mathfrak{g} \otimes$ $\left.S / I_{i} S\right)^{\Gamma}$ is isomorphic to $\mathcal{L} / I_{i} \mathcal{L}$, so the induced projection

$$
\mathcal{L} \rightarrow\left(\mathcal{L} / I_{1} \mathcal{L}\right) \times \cdots \times\left(\mathcal{L} / I_{r} \mathcal{L}\right)
$$

is surjective. But

$$
I_{i} \mathcal{L}=\left(M_{i} \cap R\right) \mathcal{L} \subseteq \mathfrak{g} \otimes\left(M_{i} \cap R\right) S \subseteq \mathfrak{g} \otimes M_{i}=\operatorname{ker}\left(\mathrm{ev}_{M_{i}}\right)
$$

so

$$
\operatorname{ev}_{M}: \mathcal{L} \rightarrow \operatorname{ev}_{M_{1}}(\mathcal{L}) \oplus \cdots \oplus \operatorname{ev}_{M_{r}}(\mathcal{L})=\mathfrak{g}^{M_{1}} \oplus \cdots \mathfrak{g}^{M_{r}}
$$

is surjective, and the module $V_{1} \otimes \cdots \otimes V_{r}$ is irreducible. Tensoring with a 1-dimensional module $W$ does not change irreducibility, so $V=W \otimes V_{1} \otimes \cdots \otimes V_{r}$ is also irreducible.

Theorems 3.1 and 3.2 give a complete list of the finite-dimensional simple modules for any twisted current algebra $\mathcal{L}$. However, many of these representations are isomorphic, so we now work at classifying such modules up to isomorphism.

In Section 2, we introduced an evaluation map $\operatorname{ev}_{M}$ associated with any maximal ideal $M$ of $S$ :

$$
\begin{aligned}
\operatorname{ev}_{M}: \operatorname{Aut}_{k-L i e}(\mathfrak{g} \otimes S) & \rightarrow \operatorname{Aut}_{k-L i e}(\mathfrak{g}) \\
\psi & \mapsto \psi(M),
\end{aligned}
$$

where $\psi(M) x:=(\psi(x \otimes 1))(M)$. Through a small abuse of notation, we associated an automorphism $\gamma(M)$ of $\mathfrak{g}$ with each $\gamma \in \Gamma$ and $M \in \operatorname{Max} S$. Since $\Gamma$ (and thus $\Gamma^{M}$ ) is finite, there are finitely many $\gamma(M)$ for any fixed $M$, so the $\Gamma^{M}$-fixed subalgebra $\mathfrak{g}^{M}$ is 
reductive by [6, $\S 1.5$ Proposition 14]. The map $\Gamma \rightarrow \operatorname{Aut}_{k-L i e}(\mathfrak{g})$ induced by $\mathrm{ev}_{M}$ is not a group homomorphism. However, the group $\Gamma$ acts on $\operatorname{Aut}_{k-L i e}(\mathfrak{g} \otimes S)$ as in (2.6):

$$
{ }^{\gamma} \psi=(1 \otimes \gamma) \circ \psi \circ\left(1 \otimes \gamma^{-1}\right)
$$

for $\gamma \in \Gamma$ and $\psi \in \operatorname{Aut}_{k-L i e}(\mathfrak{g} \otimes S)$, and with respect to this action, it is easy to verify that the evaluation maps $\mathrm{ev}_{M}$ give local twisted actions of $\Gamma$ on $\mathfrak{g}$. That is, as automorphisms of $\mathfrak{g}$,

$$
\operatorname{ev}_{M}(\gamma \eta)=\operatorname{ev}_{M}(\gamma) \circ \operatorname{ev}_{M}\left({ }^{\gamma} \eta\right)
$$

for all $\gamma, \eta \in \Gamma$ and $M \in \operatorname{Max} S$. It is then straightforward to verify that

$$
\begin{aligned}
(\gamma(M))^{-1} & =\gamma^{-1}\left({ }^{\gamma^{-1}} M\right), \\
\gamma^{-1}(M) & =\left(\gamma\left({ }^{\gamma} M\right)\right)^{-1}, \\
\left({ }^{\gamma} \eta\right)\left({ }^{\gamma} M\right) & =\eta(M),
\end{aligned}
$$

for all $\gamma, \eta$, and $M$.

Lemma 3.8 Let $\gamma \in \Gamma$ and $M \in \operatorname{Max} S$. Then $\mathfrak{g}^{M}=\gamma^{-1}(M) \mathfrak{g}^{\gamma}$.

Proof Let $\mu \in \Gamma^{\gamma} M$ and $x \in \mathfrak{g}^{M}$. Then $\gamma^{-1} \mu \gamma \in \Gamma^{M}$, so $x=\left(\gamma^{-1} \mu \gamma\right)(M) x$. By (3.4), $\left(\gamma^{-1} \mu \gamma\right)(M)=\gamma^{-1}(M)^{\gamma^{-1}} \mu(M)^{\gamma^{-1} \mu} \gamma(M)$, so by (3.7) we have

$$
\begin{aligned}
\left(\gamma^{-1}(M)\right)^{-1} x & =\gamma^{-1} \mu(M)^{\gamma^{-1} \mu} \gamma(M) x \\
& =\mu\left({ }^{\gamma} M\right) \gamma\left({ }^{\mu^{-1}} M\right) x .
\end{aligned}
$$

But $\mu \in \Gamma^{\gamma} M$, so $\mu^{-1} \in \Gamma^{\gamma} M$, and thus

$$
\begin{aligned}
\left(\gamma^{-1}(M)\right)^{-1} x & =\mu\left({ }^{\gamma} M\right) \gamma\left({ }^{\gamma} M\right) x \\
& =\mu\left({ }^{\gamma} M\right)\left[\gamma^{-1}(M)\right]^{-1} x,
\end{aligned}
$$

by (3.6). Therefore, $\left(\gamma^{-1}(M)\right)^{-1} x \in \mathfrak{g}^{\gamma} M$ and

$$
\mathfrak{g}^{M} \subseteq \gamma^{-1}(M) \mathfrak{g}^{\gamma} M
$$

Replacing $\gamma$ by $\gamma^{-1}$, and then $M$ by ${ }^{\gamma} M$ in (3.9), we obtain: $\mathfrak{g}^{\gamma} \subseteq \subseteq \gamma\left({ }^{\gamma} M\right) \mathfrak{g}^{M}$. The reverse inclusion to (3.9) then follows from (3.6).

The finite-dimensional simple $\mathcal{L}$-modules are precisely the tensor products of 1 dimensional $\mathcal{L}$-modules with irreducible representations of finite-dimensional semisimple quotients of $\mathcal{L}$. In Theorem 3.1, we saw that the latter factor through evaluation maps $\mathcal{L} \stackrel{\mathrm{ev}_{M}}{\rightarrow} \mathfrak{g}^{M_{1}} \oplus \cdots \oplus \mathfrak{g}^{M_{r}}$, for some maximal ideals $M_{1}, \ldots, M_{r}$ of $S$ from distinct $\Gamma$-orbits. Pullbacks of $\mathfrak{g}^{M_{1}} \oplus \cdots \oplus \mathfrak{g}^{M_{r}}$-modules are called evaluation representations of $\mathcal{L}$. To classify the finite-dimensional simple $\mathcal{L}$-modules up to isomorphism, it thus suffices to classify irreducible evaluation representations up to isomorphism and determine which ones appear as pullbacks of representations of semisimple quotients of $\mathcal{L}$. 
Let $\operatorname{Rep}\left(\mathfrak{g}^{M}\right)$ be the set of isomorphism classes of finite-dimensional irreducible representations of the (reductive) Lie algebra $\mathfrak{g}^{M}$. Consider the fibre bundle

$$
\mathcal{B}=\coprod_{M \in \operatorname{Max} S} \operatorname{Rep}\left(\mathfrak{g}^{M}\right) \rightarrow \operatorname{Max} S,
$$

where the fibre over $M \in \operatorname{Max} S$ is $\operatorname{Rep}\left(\mathfrak{g}^{M}\right)$. The class [1] of the trivial 1-dimensional module $\mathbb{1}$ is a member of each fibre. For any section $\psi: \operatorname{Max} S \rightarrow \mathcal{B}$ of this bundle, we define the support of $\psi$ to be the set

$$
\operatorname{supp} \psi=\{M \in \operatorname{Max} S: \psi(M) \neq[\mathbb{1}]\} .
$$

A section $\psi$ is finitely supported if $\operatorname{supp} \psi$ is of finite cardinality.

By Lemma 3.8, $(\gamma(M))^{-1} \mathfrak{g}^{M}=\mathfrak{g}^{\gamma^{-1}} M$ for all $\gamma \in \Gamma$ and $M \in \operatorname{Max} S$. We write $\psi\left(\gamma^{-1} M\right) \circ(\gamma(M))^{-1}$ for the isomorphism class $\left[\phi \circ(\gamma(M))^{-1}\right] \in \operatorname{Rep}\left(\mathfrak{g}^{M}\right)$, where $\phi$ is any representative of the isomorphism class $\psi\left(\gamma^{-1} M\right) \in \operatorname{Rep}\left(\mathfrak{g}^{\gamma^{-1} M}\right)$.

Lemma 3.10 The group $\Gamma$ acts on the set $\mathcal{F}$ of finitely supported sections of $\mathcal{B}$ by

$$
(\gamma \cdot \psi)(M)=\psi\left(\gamma^{-1} M\right) \circ(\gamma(M))^{-1}
$$

for all $\gamma \in \Gamma, M \in \operatorname{Max} S$, and $\psi \in \mathcal{F}$.

Proof This is a straightforward verification.

Let $\psi$ be an element of $\mathcal{F}^{\Gamma}$, the set of finitely supported sections fixed under the action (3.11):

$$
\psi \in \mathcal{F}^{\Gamma}=\{\psi \in \mathcal{F}: \gamma \cdot \psi=\psi \text { for all } \gamma \in \Gamma\} .
$$

We now establish a bijection $T$ between $\mathcal{F}^{\Gamma}$ and the set $\mathcal{S}$ of isomorphism classes of irreducible evaluation representations of $\mathcal{L}$. For each $\psi \in \mathcal{F}^{\Gamma}$, we see that $\operatorname{supp} \psi$ is a union of (finitely many) distinct $\Gamma$-orbits $\Omega_{1}, \ldots, \Omega_{r}$ in Max $S$. Choose $\left\{M_{1}, \ldots, M_{r}\right\}$ a set of representatives of these orbits. Consider the isomorphism class $\left[\rho_{\psi}\right]$ of the representation

$$
\rho_{\psi}: \mathcal{L} \stackrel{\mathrm{ev}_{M}}{\longrightarrow} \mathfrak{g}^{M_{1}} \oplus \cdots \oplus \mathfrak{g}^{M_{r}} \stackrel{\left(\rho_{1}, \ldots, \rho_{r}\right)}{\longrightarrow} \text { End }\left(V_{1} \otimes \cdots \otimes V_{r}\right),
$$

where $\left(V_{i}, \rho_{i}\right)$ is a representative of the isomorphism class $\psi\left(M_{i}\right) \in \operatorname{Rep}\left(\mathfrak{g}^{M_{i}}\right)$.

Lemma 3.12 For every $\psi \in \mathcal{F}^{\Gamma}$, the isomorphism class $\left[\rho_{\psi}\right]$ depends only on $\psi$ and is independent of the choice of representatives $M_{i}$ and $\left(V_{i}, \rho_{i}\right)$ for all $i$. That is, there is a well-defined map $T: \mathcal{F}^{\Gamma} \rightarrow \mathcal{S}$ given by $T(\psi)=\left[\rho_{\psi}\right]$.

Proof Suppose $M_{i}^{\prime}$ is a representative of the same $\Gamma$-orbit as $M_{i}$ for some $i$. Then $M_{i}^{\prime}={ }^{\gamma} M_{i}$ for some $\gamma \in \Gamma$, and $\rho_{i} \circ \mathrm{ev}_{M_{i}}: \mathcal{L} \rightarrow$ End $V_{i}$ and $\rho_{i}^{\prime} \circ \mathrm{ev}_{M_{i}^{\prime}}: \mathcal{L} \rightarrow$ End $V_{i}$ 
determine exactly the same representation, where $\rho_{i}^{\prime}=\rho_{i} \circ \gamma^{-1}\left(M_{i}\right)$. Indeed, for all $z=\sum_{j} x_{j} \otimes s_{j} \in \mathcal{L}$, we have

$$
\begin{aligned}
\left(\gamma^{-1}\left(M_{i}\right) \circ \mathrm{ev}_{M_{i}^{\prime}}\right)(z) & =\gamma^{-1}\left(M_{i}\right) \sum_{j} s_{j}\left(M_{i}^{\prime}\right) x_{j} \\
& =\gamma^{-1}\left(M_{i}\right) \sum_{j} \gamma^{-1} s_{j}\left(M_{i}\right) x_{j} \\
& =\sum_{j} \gamma^{-1} s_{j}\left(M_{i}\right)^{\gamma^{-1}}\left(x_{j} \otimes 1\right)\left(M_{i}\right) \\
& =\sum_{j}\left(\gamma^{-1} s_{j} u_{\gamma^{-1}}\left(x_{j} \otimes 1\right)\right)\left(M_{i}\right) \\
& =\sum_{j} u_{\gamma^{-1}}\left(x_{j} \otimes \gamma^{-1} s_{j}\right)\left(M_{i}\right) \\
& =\gamma^{-1}\left(\sum_{j} x_{j} \otimes s_{j}\right)\left(M_{i}\right) \\
& =\gamma^{-1} z\left(M_{i}\right) \\
& =z\left(M_{i}\right) \\
& =\operatorname{ev}_{M_{i}}(z) .
\end{aligned}
$$

(since $\left.z \in \mathcal{L}=(\mathfrak{g} \otimes S)^{\Gamma}\right)$

Hence, as maps on $\mathcal{L}$,

$$
\begin{aligned}
\rho_{i} \circ \mathrm{ev}_{M_{i}} & =\rho_{i} \circ \gamma^{-1}\left(M_{i}\right) \circ \mathrm{ev}_{M_{i}^{\prime}} \\
& =\rho_{i}^{\prime} \circ \operatorname{ev}_{M_{i}^{\prime}},
\end{aligned}
$$

and $T$ is well-defined.

Proposition 3.13 The map $T: \mathcal{F}^{\Gamma} \rightarrow \mathcal{S}$ is a bijection.

Proof By Theorem 3.1, $T$ is surjective. It is straightforward to verify that $T$ is also injective, using the argument in [16, Proposition 4.15].

Theorem 3.14 The isomorphism classes of finite-dimensional simple $\mathcal{L}$-modules are in natural bijection with the pairs $(\lambda, \psi) \in \mathcal{L}^{*} \times \mathcal{F}^{\Gamma}$, such that $\lambda$ vanishes on $[\mathcal{L}, \mathcal{L}]$, $\mathcal{L} / \operatorname{ker}\left(\rho_{\psi}\right)$ is semisimple, and $\operatorname{ker}\left(\lambda+\rho_{\psi}\right)=\operatorname{ker}(\lambda) \cap \operatorname{ker}\left(\rho_{\psi}\right)$ for any $\rho_{\psi} \in T(\psi)$.

Proof By the argument in Theorem [3.1, every finite-dimensional simple $\mathcal{L}$-module $(U, \phi)$ is the pullback of a representation $W \otimes V$ of a (reductive) Lie algebra $\overline{\mathcal{L}}=$ $\mathcal{L} / \operatorname{ker} \phi=Z \oplus L$, where $Z$ is the centre and $L$ is the derived subalgebra of $\overline{\mathcal{L}}$. Here $W$ is a 1-dimensional $Z$-module, on which $Z$ acts by scalars determined by a linear functional $\lambda \in Z^{*}$. Since $Z=(Z \oplus L) / L=\overline{\mathcal{L}} /[\overline{\mathcal{L}}, \overline{\mathcal{L}}] \cong \mathcal{L} /([\mathcal{L}, \mathcal{L}]+\operatorname{ker} \phi)$, such $\lambda$ may be identified with linear functionals on $\mathcal{L}$, which vanish on $[\mathcal{L}, \mathcal{L}]+\operatorname{ker} \phi$. 
By Proposition 3.13, the $\mathcal{L}$-module $(V, \rho)$ obtained by pulling back the $L$-action on $V$ to $\mathcal{L}$ is then described, up to isomorphism, by a $\Gamma$-invariant map $\psi \in \mathcal{F}^{\Gamma}$. It is clear that $\operatorname{ker} \rho$ is an ideal of $\mathcal{L}$ which contains both $\operatorname{ker} \phi$ and the preimage $\mathcal{Z} \subseteq \mathcal{L}$ of $Z$ under the projection $\mathcal{L} \rightarrow \overline{\mathcal{L}}$. That is, $\mathcal{L} / \operatorname{ker} \rho$ is a quotient of $L$, a semisimple Lie algebra, so $\mathcal{L} / \operatorname{ker} \rho$ is semisimple. Moreover, $W$ is 1 -dimensional, so $\operatorname{ker} \phi=\operatorname{ker}(\lambda+\rho)$. Thus $\operatorname{ker}(\lambda) \supseteq \operatorname{ker}(\lambda+\rho)$, from which it follows that $\operatorname{ker}(\lambda+\rho)=\operatorname{ker}(\lambda) \cap \operatorname{ker}(\rho)$. Conversely, any pair $(\lambda, \psi)$ satisfying the conditions of the theorem will determine a finite-dimensional irreducible representation $(W \otimes V, \phi)$, where $\lambda$ determines a representation $W$ of the centre of $\overline{\mathcal{L}}=\mathcal{L} / \operatorname{ker} \phi$ and $\psi$ gives a representation $V$ of its derived subalgebra $[\overline{\mathcal{L}}, \overline{\mathcal{L}}]$.

Suppose the representations $(W \otimes V, \phi)$ and $\left(W^{\prime} \otimes V^{\prime}, \phi^{\prime}\right)$ determined by two such pairs $(\lambda, \psi)$ and $\left(\lambda^{\prime}, \psi^{\prime}\right)$ are isomorphic $\mathcal{L}$-modules. Then $\operatorname{ker} \phi=\operatorname{ker} \phi^{\prime}$ and the factorizations $W \otimes V$ and $W^{\prime} \otimes V^{\prime}$ correspond to the same (unique) decomposition $\overline{\mathcal{L}}=$ $\mathcal{L} / \operatorname{ker} \phi=Z \oplus L$ described above. The representations $W \otimes V$ and $W^{\prime} \otimes V^{\prime}$ are isomorphic as $\overline{\mathcal{L}}$-modules, and by [3, $\S 7.7$ Proposition 8], $W \cong W^{\prime}$ as $Z$-modules and $V \cong V^{\prime}$ as $L$-modules. By the discussion above, we then see that $\lambda=\lambda^{\prime}$. Similarly, the pullbacks of $V$ and $V^{\prime}$ to $\mathcal{L}$ are isomorphic $\mathcal{L}$-modules, so $\psi=\psi^{\prime}$ by Proposition 3.13 .

\section{References}

[1] J. Auger and M. Lau, Extensions of modules for twisted current algebras, in preparation.

[2] P. Batra, Representations of twisted multi-loop Lie algebras, J. Algebra 272 (2004), 404-416.

[3] N. Bourbaki, Éléments de mathématique: Algèbre, Chapitre 8, Hermann, Paris, 1958.

[4] N. Bourbaki, Éléments de mathématique: Algèbre commutative, Chapitre 5, Hermann, Paris, 1964.

[5] N. Bourbaki, Éléments de mathématique: Groupes et algèbres de Lie, Chapitre 1, Hermann, Paris, 1972.

[6] N. Bourbaki, Éléments de mathématique: Groupes et algèbres de Lie, Chapitres 7 et 8, Hermann, Paris, 1975.

[7] V. Chari, Integrable representations of affine Lie-algebras, Invent. Math. 85 (1986), 317-335.

[8] V. Chari and A. Pressley, Integrable representations of twisted affine Lie algebras, J. Algebra 113 (1988), 438-464.

[9] V. Chari, G. Fourier, and T. Khandai, A categorical approach to Weyl modules, Transform. Groups 15 (2010), 517-549. 
[10] E. Date and S.-s. Roan, The structure of quotients of the Onsager algebra by closed ideals, J. Phys. A 33 (2000), 3275-3296.

[11] K. Erdmann and M. Wildon, Introduction to Lie Algebras, Springer Undergrad. Math. Ser., Springer, London, 2006.

[12] B. Feigin and S. Loktev, Multi-dimensional Weyl modules and symmetric functions, Comm. Math. Phys. 251 (2004), 427-445.

[13] B. Hartwig, The tetrahedron algebra and its finite-dimensional irreducivle modules, Linear Algebra Appl. 422 (2007), 219-235.

[14] M. Lau, Representations of multiloop algebras, Pacific J. Math. 245 (2010), 167184.

[15] M. Lau and A. Pianzola, Maximal ideals and representations of twisted forms of algebras, Algebra Number Theory 7 (2013), 431-448.

[16] E. Neher, A. Savage, and P. Senesi, Irreducible finite-dimensional representations of equivariant map algebras, Trans. Amer. Math. Soc. 364 (2012), 2619-2646.

[17] S.E. Rao, On representations of loop algebras, Comm. Algebra 21 (1993), 21312153.

[18] S.E. Rao, Classification of irreducible integrable modules for multi-loop algebras with finite-dimensional weight spaces, J. Algebra 246 (2001), 215-225. 